\title{
Modifiability of the serial position curve in immediate recall
}

\author{
JEAN DREVENSTEDT, Ohio University, \\ Athens, Ohio 45701
}

Forty $S s$ were tested for immediate recall of a supra-span list, subdivided by temporal and spatial cues, under conditions requiring recalls of all items and selected portions of items. An overall bow-shaped serial position curve obtained from total recalls revealed a mild intralist bowing pattern consonant with induced sublists, but further indicated pronounced $S$ variability in list and sublist anchoring effects. While data indicated cues were effective in promoting list division into prescribed sublists, no support was found for an interaction effect between facilitated recall of sublist units and use of sublist anchor points when Ss retrieved entire lists.

Studies have demonstrated that the characteristic bowed shape of the serial-position curve in serial-learning performance can be modified by experimental instructions to Ss and also by certain attention-directing cues (Feigenbaum \& Simon, 1962; Glanzer \& Dolinsky, 1965; Reynolds \& Houston, 1964; Wishner, Shipley, \& Hurvich, 1957). For example, distinguishable sublists within the series of items to be learned can change the typical bowed curve, presumably by introducing anchor points into the list. Wishner et al (1957) found that, by inserting cues into a list of nonsense syllables by which the main list could presumably be divided in to two or three sublists, serial-position curves were doubly or triply bowed, whereas control lists showed a systematic pattern of bowing. The investigators presented an account of serial learning that hypothesized that $S s$ attempt to find anchoring points in a list. Glanzer \& Dolinsky (1965) have further suggested that Ss may make choices of cues by which to anchor serial-position curves in rote learning, and that any cue may serve as an anchor for the curve.

The effects of experimentally induced cues upon serial-position curves have typically been derived from analyses of group data in serial-learning tasks using an anticipation method of responding. The present study was concerned both with generalizing to an immediate recall task the effectiveness of sublist terminal points in modifying the serial-position curve and with assessing $S$ variability in the use of such terminal points. In recall of a perceptually subdivided supra-span list, Ss might be hypothesized to make differential use of terminal positions as anchors, consistent with the unitizing strategies by which they attempt to organize the memory task. Some Ss, for instance, might unitize the list as one long interconnected sequence of items; others, as a succession of shorter lists, if they indeed attend to cues dividing the series; and still others, perhaps as subdivisions within a main list. Thus, potential anchor points would be available at both ends of the sublists, as well as both ends of the entire lists, although Ss might well differ in the extent to which such cues acquired uniqueness as terminal positions and thus provided potential anchors for the recall task. To the degree that analyses of overall group data obscure such differences, little information typically is provided about $S$ variability in the effectiveness of such anchoring cues.

The reported study was designed (1) to demonstrate, in an immediate recall task using a modified free-recall method, S variability in the use of list and sublist terminal positions as anchoring points; and (2) to explore the relationship between $\mathrm{Ss}^{\prime}$ use of sublist cues in total recalls and their accuracy in retrieving the sublists, which might support a hypothesis relating $\mathrm{Ss}^{\prime}$ unitizing strategies in organizing the task and the shape of the subsequent serial-position curve upon total recall.

\section{SUBJECTS}

The $S s$ were 40 undergraduate women students who participated for extra course credits in introductory psychology classes. No $S$ had previously taken part in a memory experiment of any kind.

\section{APPARATUS AND STIMULI}

The item-presentation board was an $8 \times 8$ in. panel containing nine tubular apertures, each $13 / 4$ in. in diam. [Refer to Drevenstedt (1966) for details of a slightly modified apparatus.] Apertures were aligned $7 / 8$ in. apart in three horizontal rows of three cells each. Cells were successively illuminated at controlled time intervals by a stepper-relay system, beginning with the upper left-hand cell and proceeding row by row, left to right. A recall board, identical in panel design, was placed 9 in. to the left of the presentation board on a table. Individual-cell and master switches allowed $\mathrm{E}$ to light any one cell or, simultaneously, any group of cells on the recall board, with Ss' recalling the entire list or any portion of the list when $E$ illuminated the corresponding cells on the recall board. Both boards were observed by E, who was screened from Ss' view but thus maintained a verification of cue patterns and apparatus reliability.

On every presentation, each row of cells contained one digit and two consonants. Consonants were drawn from the set $B$ through $L$ (omitting $G$ ), digits from the set 2 through 9 (omitting 1). Items were selected and positioned randomly for each row, with no repetition of item on any one trial. Block-lettered, 1-in. symbols were drawn in mirror image on the reverse side of translucent sheets of paper. When a sheet was inserted into the presentation board, items became visible within cells as the cells were sequentially lighted behind them.

\section{PROCEDURE}

Each $S$ was tested individually for total recall of all nine items (TR) and for three partial-recall methods: (PR-1) recall of one row of three items, (PR-2) recall of one vertical column of three items, and (PR-3) recall of three random items from the entire list. Thirty-six trials were given-nine for each recall method, rerandomized after every fourth $S$ to reduce presentation-order effects. An equal number of trials was given for each row and each column in PR-1 and PR-2 methods. For PR-3, a sample was drawn randomly, with redrawings made, as necessary, to prevent items from occurring in spatially sequential positions and to maintain the required digit-to-consonant ratio.

The Ss completed two practice trials from each recall method before starting the experiment. On a trial, each of the nine items was shown for $1.0 \mathrm{sec}$, with the next sequential item appearing as the preceding one terminated. A 1.0 -sec temporal pause occurred at the end of each row. When a pattern of cells on the recall board was lighted $1.0 \mathrm{sec}$ after a series ended, the $S$ wrote the requested items on a scorecard. Scorecards were partitioned into nine sections, corresponding to the cells of the boards, and Ss were required to write the items in their correct spatial locations, although in any order they wished. The recall board remained lighted $30 \mathrm{sec}$, with approximately $25 \mathrm{sec}$ between trials to reduce interference effects. One 2-min rest period was allowed following Trial No. 18.

\section{RESULTS}

The quantity of items recalled correctly for each serial position under TR was summed over trials for each $S$ and expressed as a percentage of possible correct responses. When plotted, the data revealed an overall bow-shaped serial-position curve with facilitated recall for the initial and the last three items, as 
well as mildly improved recall for terminal positions of the three rows as compared with the middle item of the row.

Since characteristic primacy and recency effects on the list had been obtained, as well as a mild intralist triple-bowing effect consonant with the rows, a further analysis was attempted to show any differential performance patterns within Ss. All 40 Ss were ranked by size of absolute difference between their mean percentage score on the initial item of the list and their mean composite score on the final three items. Data of the 20\% (N=8) showing most pronounced primacy effects were averaged and plotted as Ss A (Fig. 1). Likewise, data of the $20 \% \quad(\mathrm{~N}=8)$ showing most pronounced recency effects were plotted as a separate curve, Ss B (Fig. 1). By inspection, the individual scores of the remaining 24 Ss (Ss C) revealed comparatively small differences in accuracy between end positions of the lists (range: $25.0 \%-0.0 \%$ difference). As shown in Fig. 1, subgroups demonstrated markedly dissimilar serial-position patterns, although the serial-position curve, initially averaged over all $40 \mathrm{Ss}$, had shown the characteristic primacy-recency effects usually obtained in serial tasks, modified by intralist bowing.

On TR, Ss A and B showed no significant difference in average accuracy, although both subgroups performed well below the level of Ss C (73.6\%) (Table 1). Although an analysis of variance indicated a significant superiority on partial recalls for Ss A over Ss B, this finding was difficult to interpret since subgroups had not been selected randomly from total $\mathrm{N}$, and there was no way of knowing what selection factors might have been contributing to the observed superiority. More importantly, PR-1 (75.4\%) was found to be superior across both subgroups at $p<.05 \quad(F=3.72$, with $2,28 \mathrm{df})$, when compared with accuracies on PR-2 (66.8\%) and PR-3 (66.5\%). That the horizontal rows provided effective cues for subdividing the list was further supported by the data of Ss C. Rows were also recalled by these 24 Ss significantly more accurately at $\mathrm{p}<.01$ than either PR-2 or PR-3 ( $\mathrm{t}=3.29 ; 3.01$, with $23 \mathrm{df})$, and likewise more accurately at $\mathrm{p}<.01$ than TR $(t=5.98$, with $23 \mathrm{df})$. Thus, analyses indicated that the experimentally induced

\begin{tabular}{lllll}
\multicolumn{5}{c}{ Table 1 } \\
\hline Subject & PR-1 & PR-2 & PR-3 & \multicolumn{1}{c}{ TR } \\
\hline A & $79.9 \%$ & $75.7 \%$ & $70.1 \%$ & $64.1 \%$ \\
B & 70.8 & 58.0 & 62.9 & 60.5 \\
\hline
\end{tabular}

Fig. 1. Percentage correct total recall by serial position of item for three $S$ groups showing variable primacy-recency effects. sublist rows were recalled significantly better by all three $S$ subgroups, as compared with other partial recalls of equal size or with total recalls, giving support to the effectiveness of the sublists as cues for subdividing the series.

There was no significant interaction between partial-recall methods and Ss A or B. Despite the observed intralist triple-bowing effect in the performance pattern of Ss B when recalling total items (Fig. 1), there was no supporting evidence that these Ss were unitizing the lists by row subdivisions any more effectively, relative to other possible unitizing strategies, than were Ss $A$. Nor were there any indications that Ss B recalled spatial columns (PR-2) more effectively, despite the mildly facilitated retrieval of row end positions in total recalls (Fig. 1).

\section{DISCUSSION}

Data were interpreted as supporting the contention that, even under constant test conditions where experimentally induced cues subdivide a supra-span list and provide potential anchor points, Ss will show wide individual variability in the use of such cues when they recall the list.

On the other hand, there was evidence from the across-subgroups superiority of partial recalls of rows that $S s$ had consistently been unitizing the supra-span lists according to the temporal and spatial cues provided by the experiment. For all three subgroups, recalls of the rows had been significantly more accurate than

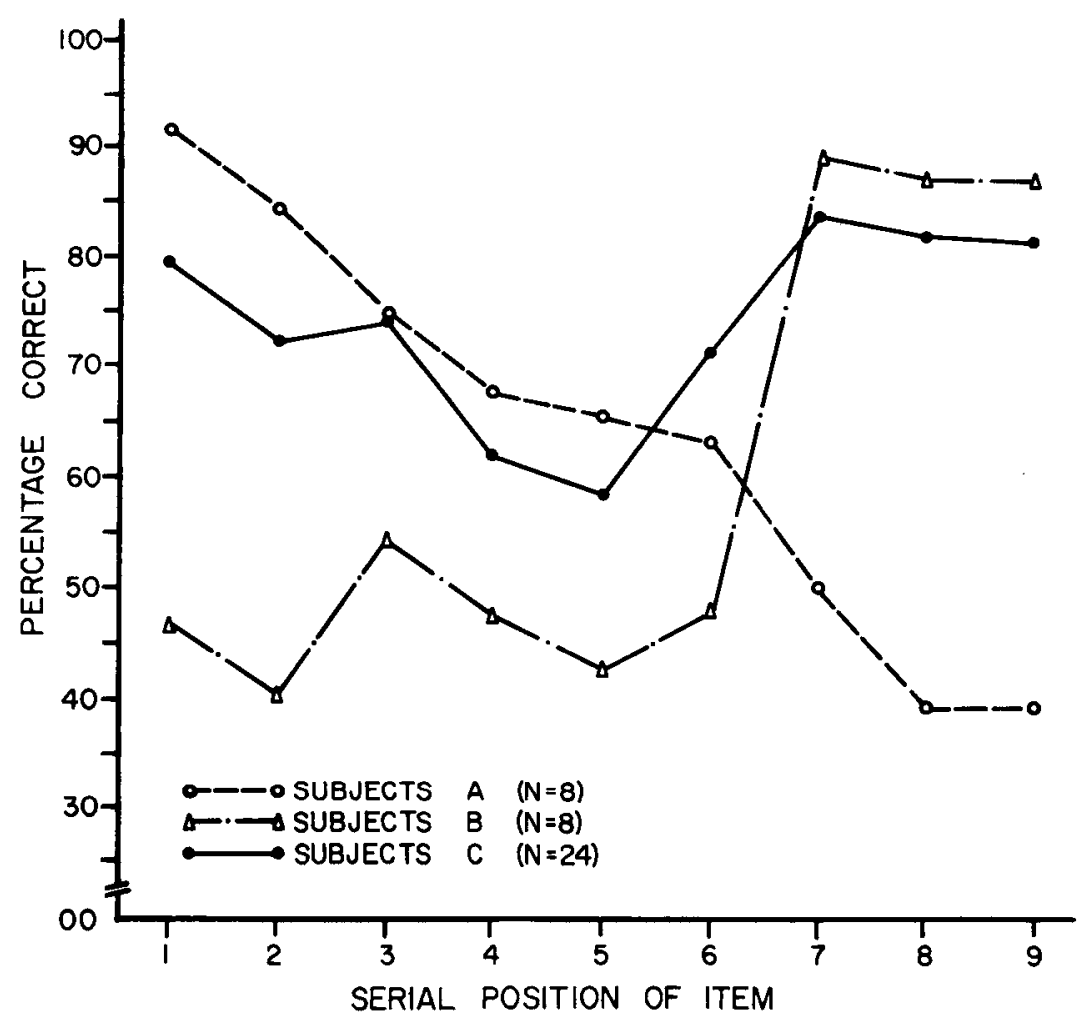

recalls of other comparable quantities of items or of entire lists. It would appear, therefore, that the discrepant patterns of serial-position performance within Ss upon total recall were not necessarily associated with different unitizing strategies Ss might have been employing to organize the lists upon their presentations. The initial hypothesis that Ss would vary in the strategies by which they attempted to organize the supra-span list in a memory task and that such variations would be reflected in differing anchor effects in recall performance was not supported.

An alternate explanation in terms of ' Ss' having anchored their recalls from various points in the list at the time of retrieval would appear more tenable. Whether these results were determined by a choice of cues as anchors through prior rehearsal emphases, or serial-order decisions at the time of report, or possibly other reasons, remains unclear. Serial-order effects were no doubt enhanced by the modified free-recall conditions of the experiment, which allowed Ss to retrieve items in any order they wished, provided locations were correct. A methodology whereby the serial order of retrieval was recorded would provide further understanding of the cues attended to first at the time of recall.

\section{REFERENCES}

DREVENSTEDT, J. Recall as a function of quantity and encoded clustering of items elicited under two methods of presentation. Journal of Experimental Psychology, 1966, 72, 551-557. 
FEIGENBAUM, E. A., \& SIMON, H. A. A theory of the serial position effect. British Journal of Psychology, 1962, 53, 307-320.

GLANZER, M., \& DOLINSKY, R. The anchor for the serial position curve. Joumal of Verbal Learning \& Verbal Behavior, 1965, 4, 267-273. REYNOLDS, J. H., \& HOUSTON, J. P. Rehearsal

\section{Range of sequential constraint in monologue rhythms ${ }^{1}$}

JOSEPH JAFFE, STEPHEN BRESKIN, and LOUIS J. GERSTMAN, ${ }^{2}$ New York State Psychiatric Institute, New York, N.Y. 10032

Using an adhoc model, previous research has found the presence or absence of speech samples taken 4 to 5 sec apart to be statistically independent. The present paper applied a chi-square test to the 5-min monologues of $50 \mathrm{Ss}$, and found the range of sequential constraint to be 2 to $3 \mathrm{sec}$. The equivalence of this result to the average breath group in acoustic phonetic research was noted.

In a previous paper from this laboratory (Jaffe, Cassotta, \& Feldstein, 1964) it was established that the time pattem of normal speech is adequately described as a two-state Markov process when presence or absence of sound is sampled at appropriate rates. ${ }^{3}$ Thus, two parameters, $q_{0}$, the conditional probability of speaking given silence in the previous sample, and $\mathrm{q}_{1}$, the conditional probability of speaking given speech in the previous sample, together specify the entire distribution of pause and vocalization durations. As the time between samples is increased, the estimates of these parameters converge upon the independent probability of vocalization so that sequential constraint is no longer detectable.

In our earlier report, we suggested that samples taken 4-5 sec apart were

Fig. 1. Cumulative percentages of talkers having converged at each sample interval under two significance criteria, $.01\left(\chi^{2}=5.412\right)$ and $.05\left(\chi^{2}=2.706\right)$. strategies and the primacy effect in seria learning. Psychonomic Science, 1964, 1 , 279-280.

WISHNER, J., SHIPLEY, T. E., \& HURVICH, M. $S$. The serial-position curve as a function of organization. American Journal of Psychology $1957,70,258-262$. given monologue and $m$ is the range of sequential constraint (in number of sample units ${ }^{4}$ ) for the criterion value of chi square with $\mathrm{df}=1$. This formula has been applied at .05 and .01 significance levels to each of 50 monologues described in our previous report, ${ }^{5}$ with results shown in Fig. 1. Independent of criterion stringency, it is observed that no Ss have converged by $0.6 \mathrm{sec}$ and that all have converged by $4.5 \mathrm{sec}$. Within that range, most converge between 1.5 and $2.7 \mathrm{sec}$, the median $\mathrm{S}$ converging at 1.8 or $2.1 \mathrm{sec}$, depending on criterion. If we take these values as our best average estimate, we arrive at the satisfying conclusion that speech samples about $2.0 \mathrm{sec}$ apart are statistically independent of each other. This corrected figure approximates the duration of an average breath group in acoustic phonetic research (Lieberman, 1967).

\section{REFERENCES}

JAFFE, J., CASSOTTA, L., \& FELDSTEIN, S. Markovian model of time patterns of speech. Science, 1964, 144, 884-886.

independent of each other, but have subsequently rejected that conclusion for two reasons: (1) The original criterion was based on an ad hoc model, and (2) the resulting estimate was not referable to the duration of any linguistic event.

Since $q_{0}$ and $q_{1}$ specify the transition matrix of a Markov process, convergence can be redefined as the first nonsignificant difference (chi-square test) between a higher power of this matrix and the matrix predicted by the independent probabilities. In addition, we have found the following direct relation between our two parameters and chi square: $\chi^{2}=\mathrm{N}\left(\mathrm{q}_{1}-\mathrm{q}_{0}\right)^{2 \mathrm{~m}}$, where $\mathbf{N}$ is the total number of samples in a

LIEBERMAN, P. Intonation, perception and language. Cambridge, Mass: M.I.T. Press, 1967 NOTES

1. We thank Professor Alex Heller of the City University of New York for the derivation of the chi-square formula

2. Also at City College of the City University of New York.

3. Analogue to digital conversion of the vocal signal at $0.3-\mathrm{sec}$ intervals was performed, with a voice relay time constant that bridged pauses less than $0.2 \mathrm{sec}$.

4. Multiplication of $\mathrm{m}$ by $0.3 \mathrm{sec}$ converts the range of sequential constraint into a time measure.

5. These were the 25 monologues reported for Condition $B$, plus 25 additional monologues for the same condition analyzed since publication.

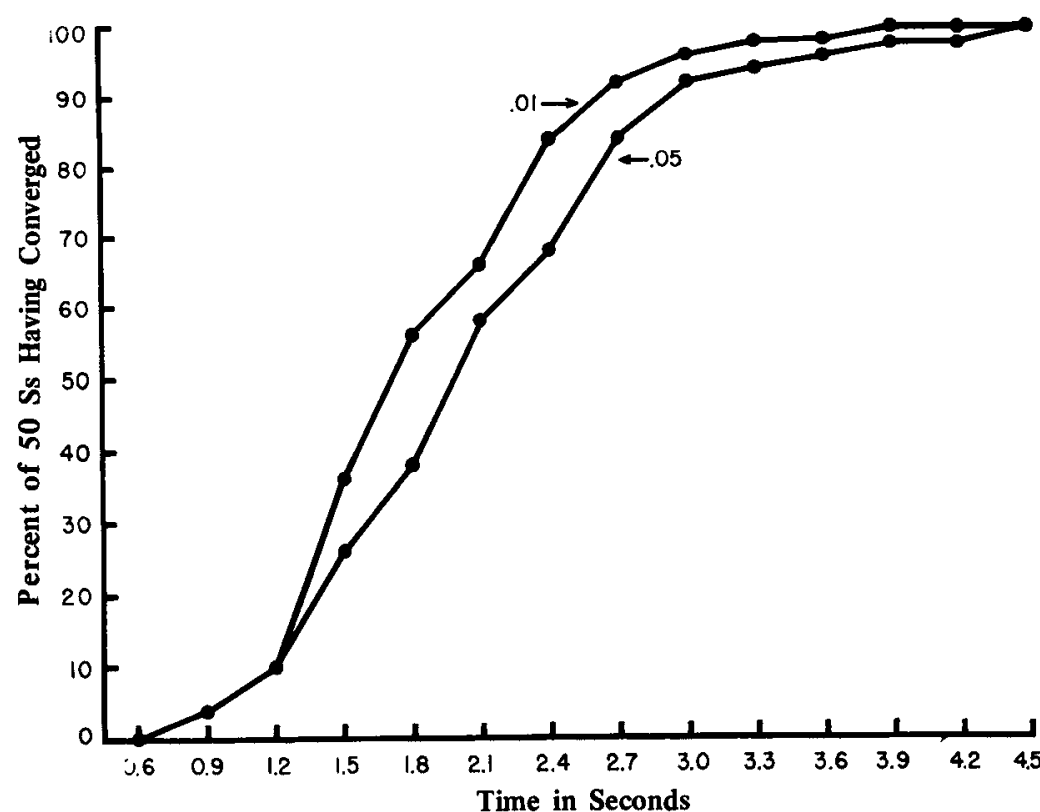

